FORMATION Formation emploi

Revue française de sciences sociales

116 | octobre-décembre 2011

Pêle-mêle

\title{
La sociologie du travail en France, enquête sur le travail des sociologues, 1950-1990
}

José Rose

\section{(2) OpenEdition}

1 Journals

Édition électronique

URL : http://journals.openedition.org/formationemploi/3485

DOI : 10.4000/formationemploi.3485

ISSN : 2107-0946

Éditeur

La Documentation française

Édition imprimée

Date de publication : 30 décembre 2011

Pagination : 69-74

ISSN : 0759-6340

\section{Référence électronique}

José Rose, «La sociologie du travail en France, enquête sur le travail des sociologues, 1950-1990»,

Formation emploi [En ligne], 116 | octobre-décembre 2011, mis en ligne le 23 janvier 2012, consulté le 30 octobre 2020. URL : http://journals.openedition.org/formationemploi/3485 ; DOI : https://doi.org/ 10.4000/formationemploi.3485

(c) Tous droits réservés 


\section{Note de Lecture}

\section{Présentation de l'ouvrage de Lucie Tanguy: La sociologie du travail en France, enquête sur le travail des sociologues, 1950-1990}

par José Rose*

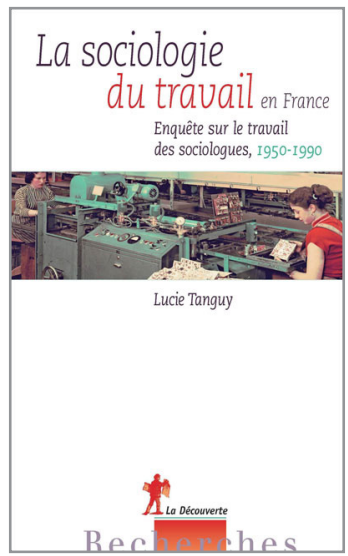

Un compte-rendu d'ouvrage peut inciter à la lecture de plusieurs façons. On peut exposer méthodiquement les idées développées au risque de réduire l'intérêt de la lecture complète, retenir simplement quelques idées au risque de s'éloigner de l'intention de l'auteur, mettre en parallèle les idées de l'auteur avec celles développées par d'autres (soi compris !) au risque d'oublier l'ouvrage dont on est censé parler. Se pose ainsi la question de la place accordée aux trois partenaires concernés : l'auteur, le commentateur et le lecteur (du commentateur et éventuellement de l'auteur !).

Dans le cas présent, on pourrait laisser la parole à l'auteure elle-même car l'introduction générale et les conclusions partielles sont particulièrement claires. Il suffirait alors de composer un montage de citations et l'objectif serait atteint. Mais on peut aussi souligner les raisons pour lesquelles on a apprécié ce livre.

*José Rose, professeur de sociologie à l'université de Provence, membre du LEST-CNRS. Thèmes des travaux : les transformations du travail, I'insertion professionnelle, les relations entre formation et emploi. Publications récentes : "Penser les catégories et leurs frontières. Travail et études : équivalence, alternance et dissonances " (in Les catégories sociales et leurs frontières, coll. s.d. A. Degenne, C. Marry et S. Moulin, Presses Universitaires de Laval, 20111 ; " Les jeunes face à l'emploi et au travail en France. Tendances structurelles et logiques d'acteurs » (in Les Jeunesses au travail. Regards croisés France-Québec, ouvrage collectif s.d. Ch Papinot et M. Vultur, Presses Universitaires de Laval, INRS, UBO, 2010I ; Les relations formation-emploi en 55 questions, ouvrage collectif coordonné avec J.J. Paul (Dunod, 2008). 
C'est ce que l'on fera en évoquant d'abord les grandes lignes de l'ouvrage puis en commentant certaines des idées avancées.

Et, avant de commencer, on pourrait mettre en exergue cette citation de Max Weber, dans Le savant et le politique : « La science met naturellement à notre disposition un certain nombre de connaissances qui nous permettent de dominer techniquement la vie par la prévision (...) elle nous apporte (...) des méthodes de pensée, c'est-à-dire des instruments et une discipline (...) elle contribue à une æuvve de clarté » (Weber M., 1919, Le savant et le politique, Editions 10/18 bibliothèques, 1959, pp. 111-112)).

\section{PRÉSENTATION DE L'OUVRAGE}

Le livre de Lucie Tanguy se donne pour objet de « reconstituer la renaissance d'une discipline scientifique et son évolution, à la lumière de faits et de données matérielles, et à distance du récit qui en a été fait et tenu pour "vrai" » (p. 8). La méthode retenue est celle du travail sur archives. "Parce que, [écrit-elle], je privilégie les faits sur les discours, les pratiques sur les énoncés généraux, la méthode inductive plutôt que les théories abstraites, j'ai entrepris de réunir un grand nombre d'informations » portant sur les politiques, les courants et les pratiques de recherche. Sont ainsi mobilisés dossiers d'acteurs de l'administration du travail, rapports de conjoncture et d'activité du CNRS, entretiens avec des chercheurs, archives du ministère de la Recherche. C'est, dit-elle encore, « un garde-fou contre les formalisations précipitées et les théories abstraites, ou simplement contre les “discours sur" » (p. 15).

La première partie est « un retour sur les commencements de la sociologie du travail en France et l'examen critique de la vision conventionnelle qui en a été donnée » (p. 15). L'émergence d'une discipline scientifique suppose la réunion de conditions institutionnelles et intellectuelles. Il s'agit donc d'examiner "les conditions sociales et politiques dans lesquelles le travail a été défini comme un domaine propre de la sociologie ». À cet égard, le rôle de l'Institut des sciences sociales du travail (ISST) ${ }^{1}$, fondé par une " conjonction d'actions émanant des milieux universitaires et politiques » (p. 29), a été plus important qu'on ne le dit généralement. Des missions de recherche envoyées par le ministère du Travail aux États-Unis revient ainsi la nécessité de combiner les disciplines et de relier enseignement et recherche pour « établir une sociologie empirique » (p. 39).

Construire une discipline passe aussi par la formation d'une communauté. À cet égard, Friedmann et Naville $^{2}$ ont joué un rôle majeur. S'ils se rejoignent sur l'idée que «le travail représente, à ce moment de l'histoire, le domaine à explorer pour rendre compte des structures sociales dans leur globalité et leur évolution» (p. 41), il vont aussi se distinguer par leur pratique, le premier s'affirmant nettement comme " entrepreneur intellectuel » prônant une recherche " empirique, concrète, de terrain et utile », cherchant le dialogue avec les responsables des entreprises et des administrations et trouvant les appuis nécessaires, tandis que le second a "une conception moins institutionnelle et plus réflexive des sciences sociales" (p. 66).

S'affirmera ainsi un «style de recherche » marqué par le traitement des questions sociales, le travail de terrain et l'analyse statistique et comparative. Nombre de recherches vont alors être financées par des organismes de productivité comme le Commissariat Général au Plan. Et l'ISST va, en dix ans, contribuer à faire exister, comme norme temporaire, " une sociologie empirique qui repose sur de grandes enquêtes visant une objectivation des phénomènes sociaux» (p. 69). Ainsi « loin de s'opposer, les recherches menées au CES et à l'ISST se sont conjuguées pour donner naissance à un style de recherche qui s 'inscrit dans un espace délimité par deux pôles : l'expertise et la critique sociale », et qui cherche à « unir intelligibilité, explication et prédiction» (p. 71).

La deuxième partie de l'ouvrage est consacrée aux années 1960-1970. Elle montre les raisons et moda-

\footnotetext{
1 Cette institution de recherche, «créée à l'initiative du ministère du Travail, rattachée à l'université de Paris et placée sous cette double autorité » a trouvé son origine dans une " conjonction d'actions émanant des milieux universitaires et politiques » (p. 29).

2 Auteurs, l'un et l'autre, d'une œuvre abondante et de travaux majeurs sur le travail, ils coopérèrent cependant le temps de faire le Traité de sociologie du travail, moment fondateur dans l'édification de ce champ scientifique (p. 65).
} 
lités de construction de cette sociologie empirique. À cet égard, les effets de contexte ont été décisifs avec "l'institutionnalisation des politiques de la science», les mesures visant le développement de la recherche, « la coopération entre chercheurs en sciences sociales et élites» (p. 79). « Suivre les actions entreprises par les chercheurs pour définir un modèle scientifique, le faire reconnaître, s'organiser en communauté, débattre des perspectives érigées en théorie et faire admettre des normes de scientificité à destination des générations suivantes », telles seront les priorités de l'époque.

«Construire une science explicative utile à l'action» va d'abord passer par une référence au modèle des sciences de la nature avec un accent mis sur la mesure et la quantification. Il s'agira alors de faire « une sociologie positive des faits sociaux» mais aussi d' « organiser la recherche en travail collectif, hiérarchisé, au sein de laboratoires » et de " produire des connaissances utiles pour moderniser la France ». Nombre de sociologues s'engageront alors pour «faire reconnaître leur discipline comme une science explicative et prédictive » et aussi «mobiliser des connaissances pour agir rationnellement sur le monde social» (p. 97).

Dans cette organisation d'une communauté intellectuelle, la Société française de sociologie $(\mathrm{SFS})^{3}$ va jouer un rôle important en prônant une professionnalisation de la profession. Ceci va être l'objet d'un large accord mais aussi de dissensions, moins sur la nécessité de professionnaliser la sociologie que sur le type de recherche à promouvoir. La sociologie empirique va ainsi se décliner différemment selon les courants politiques et idéologiques de l'époque. On a ainsi pu opposer «le rationalisme libéral de Michel Crozier », "le volontarisme d'Alain Touraine » et le «positivisme tempéré » de Raymond Boudon et constater que "l'accès au statut d'auteur majeur, à un moment donné, dépend de la légitimité acquise auprès de ses pairs (par son usage de la norme scientifique) mais aussi de sa capacité à fournir une analyse de la société comme totalité structurée » (p. 129) : le

3 «La SFS a été créée en 1962, avec l'appui de l'Association des sociologues de langue française (AISLF), pour rassembler tous les sociologues français, établir des contacts avec les spécialistes des disciplines voisines et avec les utilisateurs de sciences sociales et définir un mode d'exercice de l'activité scientifique » (p. 99). conflit chez Touraine, l'organisation chez Crozier, la négociation chez Reynaud.

La troisième partie analyse "les ruptures en matière de thématiques et de perspectives d'analyse dans les années 1980-1990». Avec l'arrivée de la gauche au pouvoir, en 1981, "la sociologie du travail se voit assigner une fonction singulière : assurer les liens entre les politiques industrielles promues et les principaux acteurs de leur mise en place, dirigeants d'entreprises et syndicats 》 (p. 151). Et «trois objectifs majeurs caractérisent la politique impulsée en 1982 : la démocratisation de la recherche, la réconciliation de la recherche fondamentale et de la recherche appliquée, la reconnaissance des sciences humaines et sociales» (p. 156). À quoi on pourrait ajouter l'« injonction d'interdisciplinarité ».

L'exemple du Programme interdisciplinaire de recherche Technologie Travail Emploi et Modes de vie (PIRTTEM) illustre bien ce dialogue entre administrateurs et ce souci de "produire des connaissances utiles » mais aussi d' « organiser un milieu scientifique ». L'analyse des articles de la revue Sociologie $d u$ Travail révèle également l'évolution des thématiques de recherche, les sociologues considérant «la variabilité de leurs objets d'investigation comme un corollaire obligé de celle du questionnement social » (p. 165).

L'auteur montre aussi le rôle des « contrats de connaissance » dans la « conversion des esprits ». Ainsi s'estompent "les frontières entre recherche et expertise » (p. 185), tandis que s'affirme « la complémentarité de deux professionnalités, celle de chercheur et celle de consultant » (p. 186) et l'intérêt des recherches réalisées en partenariat. Elle évoque également «le lancement d'une sociologie de l'entreprise », précisant d'emblée que l'intérêt pour l'entreprise n'était pas vraiment nouveau chez les sociologues du travail. Dans ce domaine, on est ainsi passé « des controverses à la coexistence pacifique » et "cette réhabilitation de l'entreprise en France a été promue par les politiques et impulsée par les institutions de recherche» (p. 217). A alors émergé « une nouvelle définition de la profession de sociologue », illustrée par des auteurs comme Crozier et Sainsaulieu ou une revue comme Sociologies pratiques. Ainsi, « au delà des rôles (homme d'études, formateur, conseiller) 
et des statuts (praticien, chercheur, universitaire) qui varient dans le temps, une constante demeure: la vocation du sociologue à être l'accompagnateur du changement à tous les niveaux et dans tous les domaines » (p. 207). Et, selon les époques, la définition s'adapte aux contextes : accumuler des connaissances, déconstruire, faire des diagnostics, apporter des réponses.

L'intervention sociologique marque une nouvelle étape en « rupture avec la simple posture compréhensive de l'observateur extérieur» (p. 215), le sociologue n'étant "pas un savant mais un expert engagé dans la transformation du monde social au moyen de méthodes éprouvées par un corpus de connaissances scientifiques ". "L'affirmation de nouveaux objets de recherche et de nouvelles théories les accompagnant, loin de relever d'évolutions internes à une discipline intellectuelle, apparaît plutôt résulter de la conjonction entre des politiques de recherche ellesmêmes étroitement liées aux politiques économiques et sociales, et des initiatives d'entrepreneurs intellectuels capables de réunir autour d'eux des réseaux, pour mettre en ouvre un programme de recherches qui est, d'emblée, traduit en termes dits théoriques, qui ne sont que des énoncés généralisants résultant de partis pris sur la réalité sociale».

En conclusion, Lucie Tanguy évoque « quelques grandes questions qui font problème dans cette spécialisation » : la professionnalisation, la cumulativité, la pluridisciplinarité et la place de la sociologie au sein des sciences sociales.

\section{DISCUSSION}

Cet ouvrage propose un vaste panorama de l'histoire d'une discipline, de ses évolutions et des controverses sur quatre décennies et l'on voit ainsi se combiner des personnes et contextes, des projets de recherche et des politiques de recherche. Ceci permet, en creux, d'éclairer le contexte actuel en soulignant l'articulation entre les exigences intellectuelles du métier de chercheur, les contextes propices au développement d'une discipline et le rôle des acteurs concernés. Resterait à approfondir la place, derrière celle occupée par les « capitaines », des équipes et de tous les cher- cheurs engagés dans cette histoire et dont plusieurs pourraient témoigner aujourd'hui.

Cette recherche est également très utile pour explorer son propre parcours et son style de recherche. Elle l'est également par l'œuvre de transmission qu'elle réalise. L'auteur se voit en effet comme « un passeur » mais, précise-t-elle, " transmettre les leçons de son expérience n'a d'intérêt que s'il se fait sur le mode d'une analyse réflexive et non sur celui du témoignage " (p. 15), position qui pourrait d'ailleurs se discuter. Ce "retour sur une expérience de chercheure » essayant tant bien que mal de "tenir ensemble une enquête de terrain et des cadres d'analyse conceptuelle» (p. 8) peut être fort utile pour les jeunes chercheurs, tant pour leur permettre de mieux maîtriser l'histoire de leur discipline que pour solidifier leurs options méthodologiques. C'est enfin utile pour tous les chercheurs qui peuvent ainsi réduire les risques d'amnésie, mieux situer leur recherche dans la dynamique de la discipline et voir d'un œil plus aiguisé ce qui peut parfois se présenter comme une nouveauté alors que des débats similaires ont eu lieu par le passé : l'exemple de la « sociologie de l'entreprise » est assez illustratif à cet égard.

L. Tanguy propose ensuite une réflexion sur le métier en mettant en avant, dans sa pratique personnelle, l'importance de la " démarche par essais et erreurs», "par induction à partir d'un penchant pour une perspective d'analyse empirique » (p. 9) et "toujours guidée et habitée par le doute » (p. 11). On retrouve ainsi certaines de ses constantes - le manque de cumulativité, le poids des instances politiques, l'importance du matériau empirique - et de sa pratique. On pense à nouveau à M. Weber quand il écrit "Seul l'être qui se met purement et simplement au service de sa cause possède une "personnalité" dans le monde de la science » (Le savant et le politique, 1959, p. 86). On retrouve aussi son intention de porter un regard nouveau sur des questions récurrentes, d'apporter des matériaux pour argumenter ses positions comme elle le fait à propos du rôle sous-estimé de l'ISST ou de la pseudo nouveauté de la sociologie de l'entreprise. « La tâche primordiale d'un professeur capable est d'apprendre à ses élèves à reconnaître qu'il y a des faits inconfortables » écrivait encore M. Weber. 
Enfin, et parce que ce compte-rendu est publié dans Formation-Emploi, revue qui a profité de longue date des contributions de Lucie Tanguy, il nous a paru intéressant de mettre en perspective cet ouvrage avec les travaux et la posture du Céreq car nombre de thèmes font écho, tels l'expertise ou la pluridisciplinarité.

L'ouvrage évoque ainsi la question des rapports entre connaissance et action et c'est une préoccupation constante du Céreq. Connaissance intellectuelle et action publique se distinguent bien sûr par leur objectif (comprendre ou transformer le réel), leur temporalité (le temps long de la réflexion et le temps cours de l'action, les ressources mobilisées (intellectuelles ou pratiques) et les acteurs concernés (les chercheurs et les décideurs). Mais une articulation entre les deux est nécessaire, la connaissance intervenant aux diverses étapes de l'action publique tant dans les phases de diagnostic que dans l'accompagnement puis dans l'évaluation.

Se pose alors la question de l'expertise. De ce point de vue, le Céreq a une posture singulière puisqu'il se présente de longue date comme un "pôle public d'expertise au service de la pluralité des acteurs de la formation et de l'emploi ». À ce titre, il produit des connaissances utiles pour éclairer les décisions des divers acteurs et contribuer à la transformation de leurs pratiques et de leurs représentations. Et cela peut aussi permettre d'éclairer les débats de société. Mais cette posture ne va pas de soi car il convient de trouver le bon équilibre entre production de données, prestation d'études, travaux de conceptualisation et de recherche, activités de transfert et de valorisation. Et cela suppose une professionnalité étendue des chargés d'études. De plus, l'activité d'expertise a sa propre logique en termes de délais, de procédures de collecte de données et de types de production. Il y a donc un travail spécifique à effectuer aux diverses étapes de chaque étude / construction du partenariat, reformulation des questions posées par les acteurs, définition conjointe des objectifs, négociation pour l'accès au terrain, accord sur les modes de restitution des résultats.

Cette réflexion sur l'expertise publique était déjà abordée par Lucie Tanguy, dans "Le sociologue et l'expert : une analyse de cas » (Sociologie du Travail, 1995, $\mathrm{n}^{\circ}$ 3). «Indissociable du développe- ment des sciences sociales elles-mêmes, l'implication des sociologues dans l'analyse, le diagnostic et la recherche de solutions à une situation jugée problématique, grâce au savoir spécialisé dont ils sont détenteurs, est déjà ancienne » écrivait-elle. Et elle soulignait la spécificité de la mission ministérielle comme «forme particulière d'expertise » consistant à « demander à une personne ou des personnes singulières d'examiner une situation qui fait problème afin d'éclairer un débat, voire d'orienter une politique » et donc de «produire des informations, des faits, des analyses utiles et légitimer des prises de position par le savoir spécialisé de l'expert ». Elle mettait ainsi en avant la tension inévitable entre légitimité scientifique et sociale et soulignait les différences entre cette activité et celle de chercheur, notamment celles liées au temps et à l'objectif car "le souci d'action l'oblige à focaliser son attention sur des aspects susceptibles d'être modifiés à court terme ».

La question était également traitée dans l'article de H. Bertrand, B. Hillau et A. Richard, « L'expertise en région : entre légitimité de la connaissance et utilité pour l'action » (Formation Emploi, 2003, $\left.\mathrm{n}^{\circ} 84\right)$. Ces auteurs du Céreq s'interrogeaient en effet sur « la posture de l'expert dans la construction et la régulation de l'action publique décentralisée » et rappellaient que "la réflexion sur l'expertise doit être menée sous le double registre de la nature de la connaissance produite et de son rapport aux pouvoirs de décision ». Ils observaient ainsi la tension présente chez les élus régionaux qui «subissent la pression des besoins d'expertise pour légitimer leur rôle » et qui « récusent ce qu'est l'expertise avec ses exigences propres, son degré d'autonomie nécessaire et sa capacité à révéler des enjeux ne correspondant pas nécessairement aux représentations que s'en fait le politique ». Le problème est que «les acteurs territoriaux, comme tous les décideurs, ont une perception et un usage instrumental des études ", utilisant les études tant pour comprendre leur domaine que pour se doter d'une légitimité et conforter leur stratégie. Et l'on voit ainsi "poindre l'inévitable conflit entre deux légitimités : celle du pouvoir et celle du savoir ». Les experts subissent ainsi une double reconnaissance : celle de leurs pairs et celle des utilisateurs de la connaissance produite. Pour cela, ils doivent faire preuve de souplesse. «Un bon expert est apprécié 
pour ses analyses, sa connaissance des terrains, des acteurs, comme pour son adaptation à leurs jeux subtils, pour la pondération adroite dont il sait faire preuve dans l'énoncé maîtrisé de ses observations et de ses conclusions ». Cette " expertise experte» est rare, souvent appréciée mais risquée. Autant de réflexions qui illustrent une possible lecture de l'ouvrage de L. Tanguy du point de vue des rapports entre producteurs et utilisateurs de connaissances.

Un dernier aspect de l'ouvrage fait nettement écho aux débats présents parmi les chercheurs et au sein du Céreq, celui de la diversification des formes de recherche. En effet, l'activité des chercheurs en sciences sociales conjugue souvent recherche théorique et appliquée, études et expertise. Ce mouvement interroge l'opposition stricte entre recherche fondamentale et appliquée - toute recherche digne de ce nom ne possède-t-elle pas une dimension fondamentale dans la mesure où elle vise à mettre à jour des processus et des schémas d'interprétation ? - et incite à parler de « recherche applicable » au sens où les résultats de la recherche sont susceptibles de contribuer à la transformation du réel.
Dans cette perspective, la recherche se mesure alors non à l'aune exclusive des chercheurs mais aussi à celle des utilisateurs. La question du chercheur n'est plus seulement celle de la rigueur et de la pertinence de ses approches mais aussi celle de son utilité sociale. "Abstenons-nous donc de nous soucier a priori de la question de la scientificité pour nous borner à formuler un problème apparemment plus modeste : à quelles conditions les productions des chercheurs en sciences humaines et sociales seraient-elles susceptibles d'intéresser leurs frères humains?" écrit A. Caillé dans La démission des clercs, la crise des sciences sociales et l'oubli du politique (1993). Citation qui fait écho à celle d'E. Durkheim : «Nous estimerions que nos recherches ne méritent pas une heure de peine si elles ne devaient avoir qu'un intérêt spéculatif » (De la division du travail social, Paris, PUF, 1967, $1^{\text {ère }}$ édition 1893).

Reste à conclure. On le fera avec L. Tanguy, qui formule, dans sa conclusion, le souhait que ses travaux fassent advenir d'autres analyses et aussi avec M. Weber pour qui "toute ouvre scientifique "achevée" n'a d'autre sens que celui de faire naître de nouvelles "questions" : elle demande donc à être “dépassée” et à vieillir » (p. 88).

\section{Référence de l'ouvrage :}

Lucie Tanguy (2011), La sociologie du travail en France, enquête sur le travail des sociologues, 19501990, La Découverte, collection « Recherches ». 